\title{
Compressed sensing based fingerprint imaging system using a chaotic model-based deterministic sensing matrix
}

Workneh Wolde Wolde ( $\square$ worknehwolde11@gmail.com )

Sharda University https://orcid.org/0000-0001-5051-2547

Pallavi Gupta

Sharda University

\section{Research Article}

Keywords: Compressed Sensing, Fingerprint Image, Sparse Representation, Convex Optimization, Encryption, Chaotic Model

Posted Date: July 26th, 2021

DOI: https://doi.org/10.21203/rs.3.rs-605923/v1

License: (c) (i) This work is licensed under a Creative Commons Attribution 4.0 International License.

Read Full License 


\title{
Compressed sensing based fingerprint imaging system using a chaotic model-based deterministic sensing matrix
}

\author{
Workneh Wolde • Pallavi Gupta
}

Received: date / Accepted: date

\begin{abstract}
A novel deterministic sensing matrix design approach applied to enable secured compressed sensing and transmission of fingerprint images. The performance of the sensing matrix was analyzed in detail by varying compressed sensing and security parameters. The number of sampling and sparse coefficient are the parameters taken under consideration from compressed sensing, whereas the encryption key is from the security scheme. The first stage in the performance study is acquiring the signal, and followed by sparsely modelling it using Orthogonal Matching Pursuit (OMP) algorithm. The sparse modelled data is multiplied by the proposed deterministic partial orthogonal Discrete Cosine Transform (DCT) sensing matrix to reduce its dimension and encrypt it. To introduce confusion on the DCT matrix rows, the pseudorandom permutation is applied to the DCT matrix rows before sensing matrix derivation. Additionally, recovering and decryption of the compressed image accomplished with the help of a convex optimization method. The results obtained from the simulation of the proposed system confirmed that a better Peak Signal to Noise Ratio (PSNR) than the recommended value for wireless transmission is archived using a sample below $25 \%$ without losing a significant number of fingerprint minutiae.
\end{abstract}

Keywords Compressed Sensing · Fingerprint Image · Sparse Representation · Convex Optimization · Encryption · Chaotic Model

Workneh Wolde

Sharda University, Greater Noida, Uttar Pradish, India

Tel.: +91-7428102445

E-mail: worknehwolde11@gmail.com

Pallavi Gupta

Sharda University, Greater Noida, Uttar Pradish, India

E-mail: pallavi.gupta2@sharda.ac.in 


\section{Introduction}

High-level security demand by various facilities, intelligent devices and internet of things IoT based communications attracted researchers' attention to work on a sensors array for imaging purposes. One of them is fingerprint scanning. In past, several design strategies has been employed and most of them were a sensors array-based includes capacitive [1][2][3][4], ultrasonic [5], and optical [6] microelectromechanical system MEMS with a whole sampling method that provides for reading and recording for all sensors' outputs. In terms of the optimal design approach, complete sampling is not an efficient one.

Designing a large sensor grid with a reduced sampling is a methodology employed to leverage those difficulty associated with signal processing parts of the previous design. The strategy used in those designs is called compressed sensing that works mainly based on the concept developed by Candes et al. [7] and Donoho [8]. This formulation allows sampling from few acquired data rather than from the whole elements in the system.

The signal must be sparse in its original or transform version to implement compressive sensing formulation on the signal processing part of a particular system. The sparsity of the signal in its transform version might come in two types. One of them is obtained over some dictionary formed analytically by DCT and wavelet transformation of itself [9][10]. The other is over some learned dictionary designed based on prior knowledge about several correlated signals [11][12][13][14][15]. Compressed sensing based on the latter design approach is less lossy and suitable for fingerprint image sampling, compression, and recognition.

Furthermore, the scope of compressed sensing is not limited by analytical computations, and there is also hardware implementation. A field-programmable gate array or FPGA device is used and shows higher performance in ECG, EEG [16][17][18] and other one-dimensional signal processing [19].

Not all but some of the current sensor array system developed by researchers are compressed samplings based. These systems, however, must always deal with sparse input signals. A sparse input signal can be recovered to its original form from few measured samples within a compressed samplingsbased signal processing system [7][20]. It can be achieved by transformation of a high dimensional signal to a lower one through matrix multiplication once the actual measurements are available from the input [21].

Transmission of an image that has a biometric feature should be secured and reliable. Therefore, a cryptosystem is securely stored for reproduction, authentication or, recognition. In the past, Pseudo-random number-based encoding methods used for purposes of data encryption. Some of them are phase encoding schemes using joint transform [22], exclusive-OR encryption [23], and 
fractional Fourier transformation methods [24]. Additionally, how to encrypt fingerprint images using orthogonal coding also studied in [25]. Double stage chaotic biometric image like fingerprint encryption scheme by using two maps named Arnold (permutation) and Henon (substitution) for pixel shuffling is studied in [26].

In the work presented in this paper, a design strategy for optimized and secured sensing and transmission system with the help of a novel proposed deterministic sensing matrix introduced. The proposed system able to compressed the acquired data but not directly save or transmit it. It performs some signal processing that allows the system to code the image if it has no control over the communication channel when it sends or shares it.

The proposed, secured, and novel compressed sensing designed approach is based on a deterministic, partially orthogonal sensing matrix whose row index re-arranged in chaotic sequence. Therefore, an algorithm that governs the construction of the proposed sensing matrix is developed, and its validity is tested by employing animage quality and security assessment techniques. The reliability and optimality of the approach also demonstrated by applying the algorithm to derive several sensing matrices from a single well-known hardware friendly DCT matrix. The overall design implementation is done with the help of MATLAB script, which runs on a personal computer.

Hence the remainder of this paper organized into seven sections, including this introduction part. Section two will discuss the theoretical background of compressed sensing, including a general introduction about data compression and recovery. Following section three, the detailed methodology of designing the whole compressing sensing and its parts like dictionary and sensing matrix with their algorithm is employed to build them. The performance of the sensing matrix is studied by varying compressed sensing and image recognition parameters in section four. The performance analysis of the system from the security point of view is presented by modelling security trait and analyzing it in section five and six. Finally, the whole work is summarized by pointing out the main contribution in section seven of the paper.

\section{Background studies}

The main idea of compressive sensing is to recover signals from fewer measurements that are less than the Nyquist rate [7][8]. In addition to the sparse input signal, the system must be stable against spatial transformation caused by the change of orientations of the input during contact between fingertip and sensor array for the successful design of a compressed sensing system. Hence, we select and incorporate the necessary background theories in this section. 


\subsection{Concept of compressed sensing}

In a compressed sensing system, sparse signal $x \in \mathbb{R}^{N \times 1}$ (column vector), measured signal $y \in \mathbb{R}^{M \times 1}$ and a sensing matrix $\Phi \in \mathbb{R}^{M \times N}$ provided that $M \ll$ $N$,related by the following equation, eq. 1

$$
y=\Phi x
$$

For a given integers $k$ and $N$ with $k<N$, the sets of $k$-sparse, $S_{k}$ vectors in $\mathbb{R}^{N}$ based on [27]define as

$$
\left.S_{k}:=\left\{x \in \mathbb{R}^{N}: \mid \operatorname{Supp}(x) \leq k\right) \mid\right\}
$$

Any system of a linear equation similar to eq. 1 is known are under-completed and will lead us to find a non-unique solution. To get a unique solution, one has to set constraint and apply it to the following optimization problems defined by $L_{P}$

$$
\begin{gathered}
L_{p}: \quad \min \|x\|_{p} \quad \text { s.t. } \hat{y}=\Phi \hat{x} \\
\|x\|_{p}=\left(\sum_{i=1}^{N} x_{i}{ }^{p}\right)^{1 / p}
\end{gathered}
$$

In both equations (3) and (4), the range of $\mathrm{p}$ can be any value between 0 and 1 , and the other acceptable deal is 2 . One must solve eq(3) to get sparse vector $\hat{x}$ from $\hat{y}$ by solving $p=0$ and $p=1$. In this project, a well-known greedy type method known as Orthogonal Matching Pursuit (OMP)[28] which $p=0$ based optimization method.

In those cases, the sparse representation $\alpha \in \mathbb{R}^{N^{\prime} \times 1}\left(N^{\prime}>N\right.$ in most cases $)$ of $x$ to satisfy the condition for compressed sensing formulation needs to be obtained. Taking all the above pre-conditions, the general equation for compressed sensing the form of eq. (5),

$$
y=\Phi x=\Phi \Psi \alpha
$$

Where, $\Psi \epsilon \mathbb{R}^{N^{\prime} \times N}$ is sparse basis or dictionary and $\alpha \in \mathbb{R}^{N^{\prime} \times 1}$ sparse representation of $x$. But this formulation directly works for communication and natural signals compressible under Fourier basis [29]. Alternatively, by allowing further signal processing, the signal can be modelled to sparse using pursuit algorithms.

\subsection{Sensing matrix}

The sensing matrix that denoted by $\Phi$ in eq.(5) in this paper is a matrix used to govern the selection of sparsely modelled signal using a predetermined order of sampling process. Random or deterministic matrix [30] can be used as a sensing matrix to solve $p=1$ by employing the interior point method. 
Deterministic sensing matrix can be generated by any hardware available for signal processing, for example, FPGA units which are configured as an LFSR (Linear Feedback Shift Register) as already done in [31] for ECG signal. Additionally, a random and deterministic matric are among the matrices that satisfy the Gaussian distribution. Owing to its low memory consumption, the deterministic matric generated by hardware such as FPGA, used as encrypting scheme for the image signal and compression as it acts as a multiplier of the sparse data. The deterministic matrix used in this work is generated from the DCT matrix whose index is arranged based on the Logistics map based chaotic model [32]. A chaotic system is a dynamic system that oscillates forever without even repeating itself or shows no tendency towards steady-state value [33].

\subsection{Algorithm to find the sparse representation of input data}

The spares solution of the input data can be obtained using the Orthogonal Matching Pursuit (OMP) algorithm at a low computational cost. OMP algorithm is a greedy type algorithm that helps to solve LP0 optimization problem given by eq. 6 .

$$
\min \|x\|_{0} \quad \text { s.t. } \quad A a=b
$$

Where $A \in \mathbb{R}^{M \times N}, b \in \mathbb{R}^{N}$ and $a \in \mathbb{R}^{M}$. Since the problem is $L P 0$ type one, its solving process starts from $b$ and then looking for a column of $A$ which is most correlated with it. Once it is known, the minimum dot product value will be taken as a reference to predict the next correlated column without repetition. According to [20], this algorithm is non-invariant under an ill-conditioned dictionary.

\subsection{Dictionary learning}

Dictionary is a rectangular matrix used to obtain and study the sparse representation of the input signal before undergoing compression. One of the widely used methods to build it is known as the $K-S V D$. This method solves optimization problem eq.(3) iteratively. For a set of signals $Z$ known as training signal with initial dictionary $D$, the coefficients sets or $X$ can be obtained by solving the problem with and suitable matching pursuit algorithm given in [36]

$$
\min \|Z-D X\|_{1}^{2} \quad \text { s.t. } \quad\left\|x_{i}\right\|_{0}<K
$$

Where $K$ is sparsity level. All of the dictionary atoms are generated by updating $S V D$ (Singular Value Decomposition) methods.

The training signals are selected based on their Structural Similarity Index $(S S I M)$ [37] which is the most fundamental image quality assessment 
method. This can be done by setting the threshold value and rejecting the signal whose structural similarity index value is far below the adjusted threshold.

The structural similarity index $(S S I M)$ of an image $(a)$ with respect to its reference image $(b)$ of sizes $(N, N)$ is

$$
\operatorname{SSIM}(a, b)=\frac{\left(2 m_{a} m_{b}+B_{1}\right)\left(2 v_{a b}+B_{2}\right)}{\left(v_{a}^{2}+v_{b}^{2}+B_{2}\right)\left(m_{a}^{2}+m_{b}^{2}+B_{1}\right)}
$$

Where $m_{a}$ and $m_{b}$ are averages, $v_{a}^{2}$ and $v_{b}^{2}$ are variances, $v_{a b}$ is covariance of $a$ and $b$ respectively. And the remaining terms $B_{1}$ and $B_{2}$ are two variables to stabilize the division with leak denominator. In this paper, image patch that contains all fingerprint features selected as a reference to select the others training image patches in order to preserve the numbers of the majority fingerprint minutiae.

\subsection{Prime-dual interior-point method}

Once the signal sparse model found, then randomly sample it and transmit to the receiver where it can be recovered back to its original form will the next task. From recovery of spare representation point of view, solving the $L P-0$ type optimization problem which is a non-deterministic polynomial hard or $N P-$ Hard problem is vital.

To leverage this hardness, transforming the problem to the $L P-1$ type is the best option. Transformation of the problem leads eq. 5 to take the form of eq. 9. In addition to the sparsity of $x$, the sensing matrix $A$ must satisfy the Restricted Isometric Property $(R I P)$ with restricted isometric constant $\delta_{s}$ which given by eq.(10) [38] to get solution for eq.(3) which is also valid for eq.9 too.

$$
\begin{gathered}
\min \|x\|_{1} \quad \text { s.t. } \quad A x=b \\
\left(1-\delta_{s}\right)\|x\|_{2}^{2} \leq\|A x\|_{2}^{2} \leq\left(1+\delta_{s}\right)\|x\|_{2}^{2}
\end{gathered}
$$

According to the prime dual method, the solution can be achieved by narrowing the dual gap between the feasible solution of the prime and dual problem. It starts from an arbitrary chosen initial point and searching and going to the direction to find the optimal solution by applying the classical Newton method [39]. Implementation of this approach is computationally possible as free software packages are already available to solve the problem iteratively.

\section{The proposed method}

The proposed method to derive the proposed sensing matrix focuses on the basic Descreat Cosine Transform (DCT) eq.(11) matrix with a chaotic modelbased row confusion feature added to it through pesudo random permutaion. Its performance is studied after the derivation compleated. 
3.1 Proposed sensing matrix design for the compressed sensing system

For sensors array composed of $M$ by $N$ elements, the locations at which measurements have to be taken are stored in the sensing matrix derived from a Discrete Cosine Transform Matrix (DCT) defined by eq.11 and 12 .

$$
\begin{gathered}
\Psi_{k}=\sum_{n=1}^{N} \psi_{n} \operatorname{Cos}\left(\frac{\pi}{N}\left(n+\frac{1}{2}\right) k\right) \quad k=1,2,3, \ldots, N \\
\Psi=\left[\begin{array}{cccc}
\Psi_{1,1} & \Psi_{1,2} & \ldots & \Psi_{1, N} \\
\Psi_{2,1} & \Psi_{2,2} & \ldots & \Psi_{2, N} \\
\cdot & \cdot & \ldots & \cdot \\
\cdot & \cdot & \ldots & \cdot \\
\cdot & \cdot & \ldots & \cdot \\
\Psi_{N, 1} & \Psi_{N, 2} & \ldots & \Psi_{N, N}
\end{array}\right]
\end{gathered}
$$

Equation 11 helps us generate the matrix whose row width is not less than $k \times \log (N)[7]$ by arranging selected rows according to the recommended sequence governed by our proposed algorithm. Unlike [45], and [47], the confusion feature was added before the derivation of the sensing matrix by swapping the rows of the DCT matrix. In comparison with those schemes, in terms of optimization, the advantage is, this approach does not require building a new matrix.

For a matrix of $N$ by $N$ size, there are $N$ ! permutation options to confuse the matrix row sequence. The actual sequence of the row of the DCT matrix can generate from the index of the Logistics map recursive equation. Logistics map is mathematically given by eq.13 with the population $z_{t+1}$ at the index $t$, which varies from 1 to the maximum row size $(N)$, the ratio of the existing population to the maximum population, $z_{t}$, and growth rate $r$.

$$
z_{t+1}=r z_{t}\left(1-z_{t}\right)
$$

The value of $r$ is any value between zero and four. The values of $z_{t+1}$ and $z_{t}$ are always between zero and one. As depicted in fig.2, the logistic map is highly chaotic if the value of $r$ is 3.57 and more. The detailed step that we followed to re-arrange the matrix rows sequence summarized in algorithm-I. The resulting row combination has $1 / N$ ! probability of existing in the random permutations.

Algorithm - I Chaotic model-based row index re-arrangement (confusion) of the DCT matrix

Input : Chaotic control parameters such as chaotic initial state $z_{0}$, growth rate $r \epsilon(3.75,4]$ and the $N$ by $N$ DCT matrix.

Output : Equivalent form of the DCT matrix . 

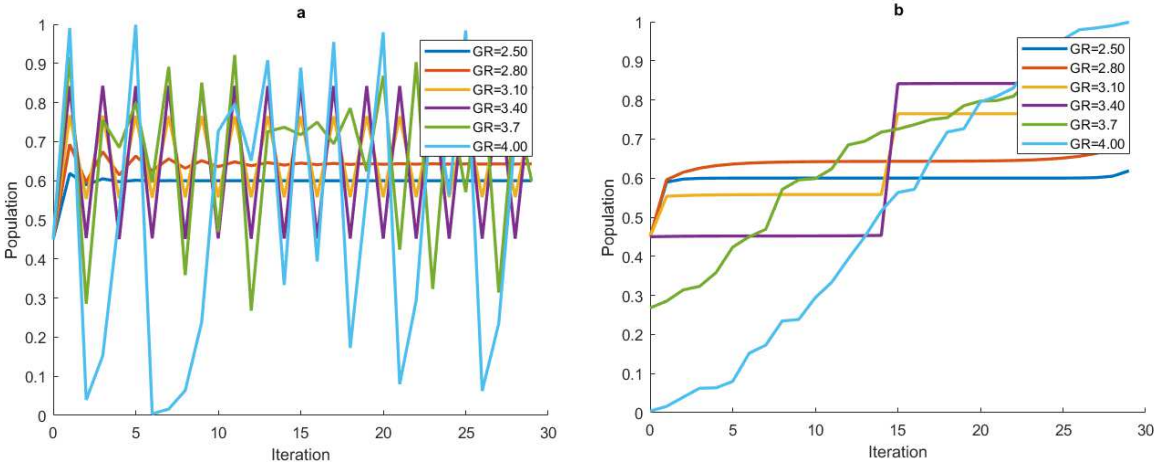

Fig. 1 Time series graph of the logistic map: (a) Standard Plot (b) Plot based on ascending order of the population

1. Using the recursive logistic map eq.(13) expression, generate a one-dimensional vector $[z[t]]$ of size $N$ by 1 .

2. Let $[N]$ be the index set $\{1,2,3, \ldots, N\}$ and their corresponding values of $[z[t]]$ are $\{z[1], z[2], z[3], \ldots, z[N]\}$

3. Performs an ascending order operation upon the $[z[t]]$ sequence which yields another new vector $[\hat{z}[t]]$ with a new index set $[\hat{N}]$.

4. Iterate through the set $[\hat{z}[t]]$ to obtain their corresponding index subset in $[N]$ and denote it by $[\mathfrak{N}]$.

5. Perform a direct mapping from set $[N]$ to $[\mathfrak{N}]$ or $([N] \leftrightarrow[\mathfrak{N}])$ and elementary rows switching of DCT accordingly.

6. Finally express the orthogonal equivalent matrix of DCT, by $\hat{\Psi}$.

The diagrammatical representation of the above algorithm has given in fig.2 for $r=3.7, t=121$ and initial state value 0.45
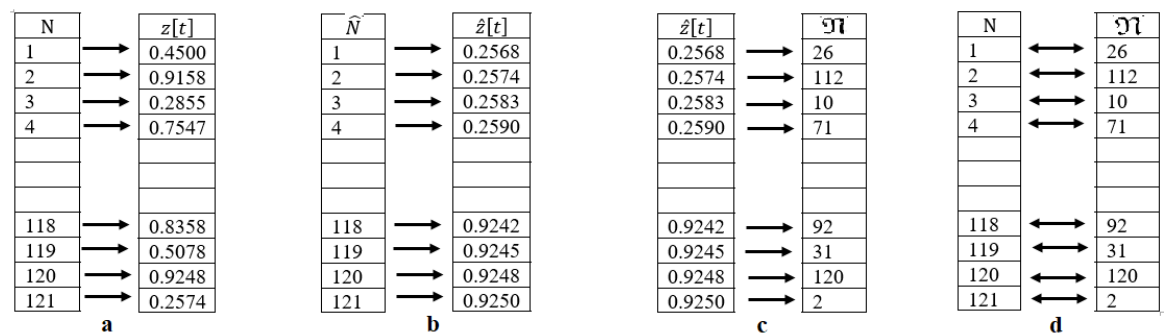

Fig. 2 DCT matrix chaotic masking process according to slgorithm-1: (a) step-1 and 2 (b) step-3 (c) step-4 and (d) step-5

Once this matrix obtained, the parameters used in this design, such as $N, r, t$, and the initial value $z_{0}$ would be taken as part of the encryption keys for the sensing system. The sensing matrix obeys the RIP, if the smallest 
set $q(M, N)$ which formed by deterministic row selection from $\hat{\Psi}$ with high probability is equal to $O(M \times \log (N))$. A Multiplicative Linear Congruential Generator (MLCG) [37] output sequence-based row selection method with slight modification can be employed to extract the sensing matrix from $\hat{\Psi}$.

The modified MLCG sequence generator has designed with shifting properties and mathematically expressed as in eq. 14 .

$$
k_{i+1}=S+\left(a k_{i}+b\right) \bmod 2^{B_{t}} \quad 0 \leq a, b \leq 2^{B_{t}-1},(M-N-1) \leq S \leq N
$$

Where $a$ is the multiplier, $b$ is the increment, $B_{t}$ is the number of bits, and $S$ is an integer used to extend the row index selection. Hence, by appropriately choosing the values $\mathrm{S}$, we can generate several sensing matrices from $\hat{\Psi}$ without repetition to make the proposed system resistive against security attack.

Algorithm - II Deterministic sensing matrix derivation

Input : Control parameters such as $S$ or the shifting value, the multipliers $a$, $b$, numbers of bit $t$, and $\hat{\Psi}$.

Output : a partial orthogonal deterministic sensing matrix $\Phi$ of $M$ by $N$ size.

1. Using the modified MLCG eq.(14) expression generate a sequence of integers $M_{\Psi}$ of size $M$ by 1 .

2. Select a sub-matrix from the equivalent form $\hat{\Psi}$ by selecting rows whose indices belongs to $M_{\Psi}$.

3. Define this sub-matrix as $\Phi$ or the deterministic sensing matrix (eq. 5 ).

4. Repeat steps 1 through 3 to generate others sensing matrices by using different parameters for MLCG.

By applying different combinations of $S, a, b$, and $B_{t}$ on the equivalent form of the $D C T$ matrix that we already at hand, we can generate many partially orthogonal sensing matrix row. Now, we can consider $S, a, b$, and $B_{t}$ as an additional encryption key for the proposed system.

Taking the outcome of the above two algorithms, the closed form of the proposed sensing matrix becomes,

$$
\Phi_{k}=\sum_{n=1}^{N} \phi_{n} \operatorname{Cos}\left(\frac{\pi}{N}\left(n+\frac{1}{2}\right) k\right) \quad k \epsilon q(M, N)=\left[M_{\Psi}\right]
$$

Each of the sensing matrix generated by algorithm-I has equal probability which is equal to $1 /(N-M)$ ! to be exist in $\hat{\Psi}$ as a sub-matrix. This is a relatively higher probability than the mentioned $1 / N$ ! as anticipated. 
3.2 The workflow to study the performance of the proposed sensing matrix

The proposed method used to examine the performance of the proposed sensing matrix is summarized in fig. 3 and 4 , which are given below

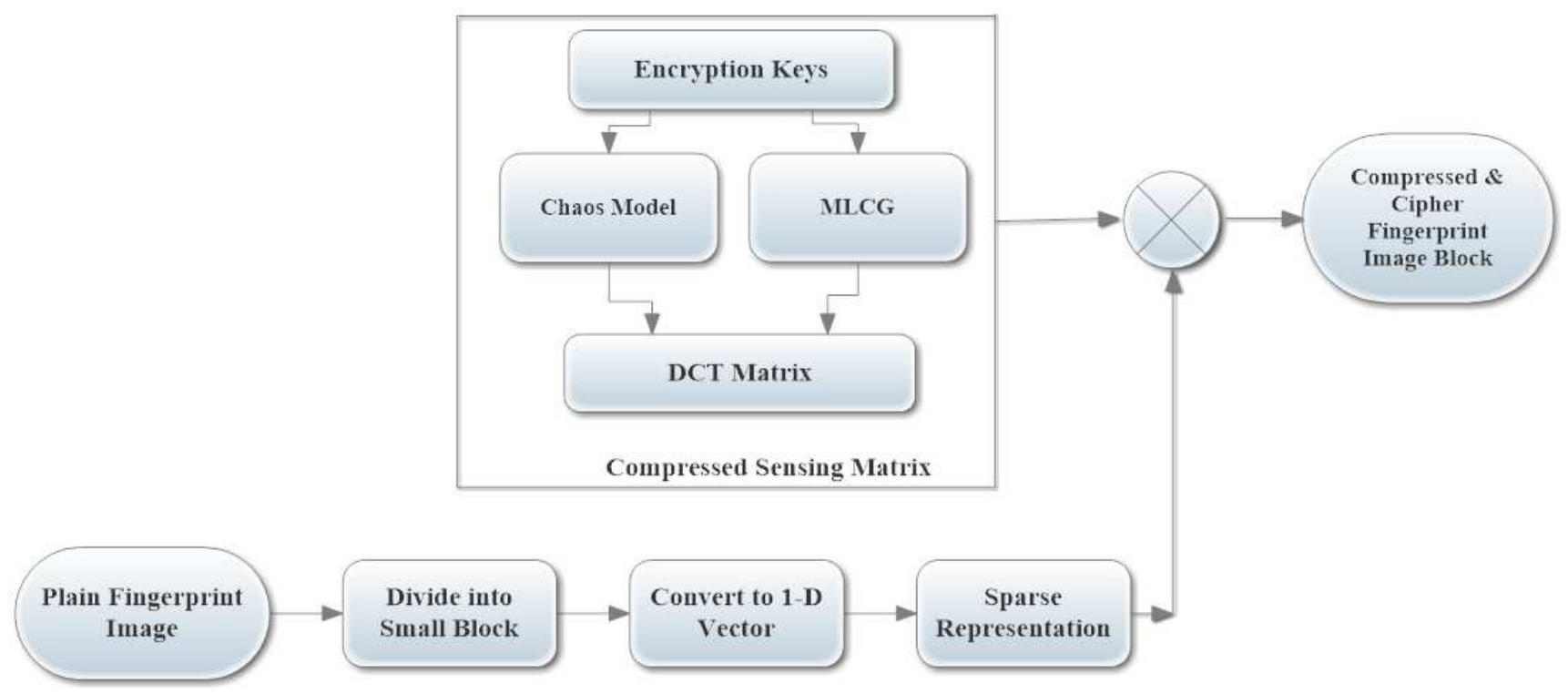

Fig. 3 The proposed compressed sensing system

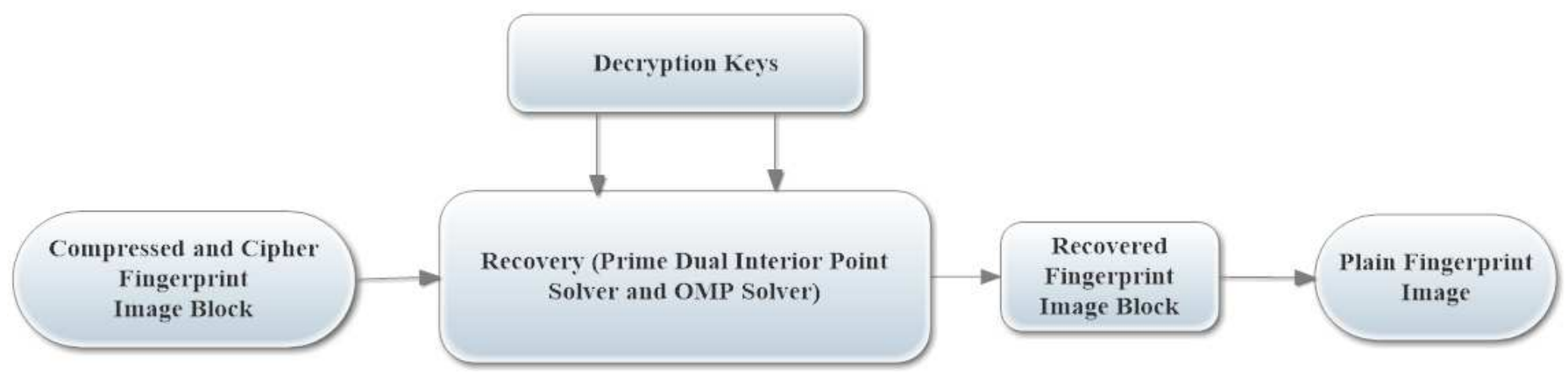

Fig. 4 The Recovery System

\section{Analysis and result for the proposed sensing matrix}

This section presents the comprehensive experimental study of the proposed system for two sensing matrices. The first one is a random matrix, and the 
other is the proposed sensing matrix. The practical test for the validity of the above numerical formulation has been done using public data sources from the NIST database (https://www.nist.gov/itl/iad/image-group/nist-specialdatabase-302).

Three assessment methods were applied apart from assessment methods employed to check the methodology's effectiveness to transform and securely transfer the input signals. These are Root Mean Square Error (RMSE), Peak Signal to Noise Ratio (PSNR), and Similarity Index of Image (SSIM). The mathematical expression for SSIM already given by eq. 8 and for the PSNR of $m$ by $n$ image with respect to reference image $a$ of a similar dimension is given by eq. 16

$$
\operatorname{PSNR}(a, b)=10 * \log \frac{(P V)^{2}}{M S E(a, b)}
$$

Where $P V$ the Peake Value of the pixel in the image and $M S E(a, b)$ is the Mean Square Error of the image with respect to reference image $a$

$$
M S E(a, b)=\frac{1}{m * n} \sum_{i=1}^{m} \sum_{j=1}^{n}(a(i, j)-b(i, j))^{2}
$$

\subsection{Dictionary learning to build the dictionary matrix}

We first organized a data store to keep a set of images which used to train our dictionary. Then we have divided each image elements to generate 8 by 8 , 16 by 16 , and 24 by 24 image patches. The similarity of each image patches is measured using their SSIM (Similarity Index) with one standard reference patch containing most fingerprint image features.

Then using the K-SVD algorithm, we trained three different dictionary matrices for signal transformation to sparse from its native form. The plot of these matrices is shown in the figure fig. 5 .
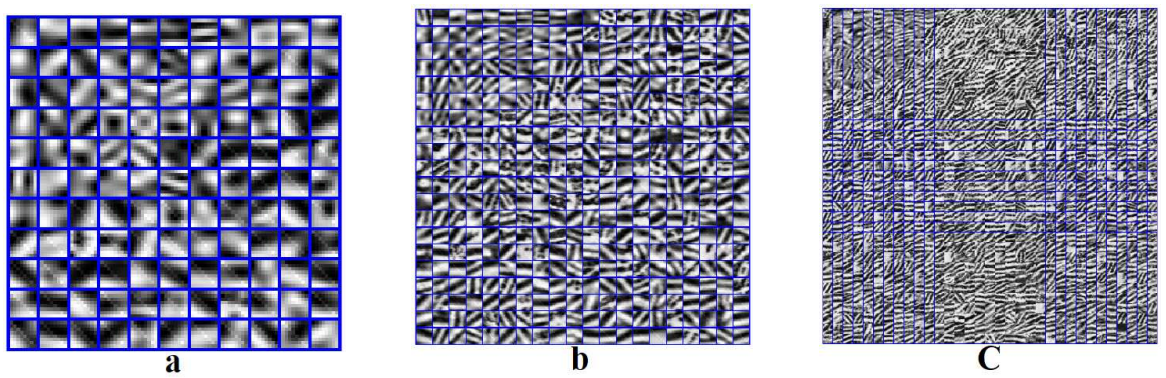

Fig. 5 The trained dictionary using (a) 8 by 8 image patches (b) 16 by 16 image patches (c) 24 by 24 image patches 
4.2 Data modeling for compressed sensing input

Using the K-SVD algorithm, we construct the dictionary matrix to model the data with length 64,256 and 576 by another equivalent representation of the input data of length 81,400 and 1089 length vector with a few numbers nonzero components, respectively. The best method to get the compressible model of this data is by employing the Orthogonal Matching Pursuit algorithm upon the measurement data.

As depicted in fig.6, our result will be better if we restrict the sparse coefficient low. This result indicates that the methodology supports a high compressional ratio like 1:10, which is pretty good in terms of the possibility of recovery. It will be proved in the next section.
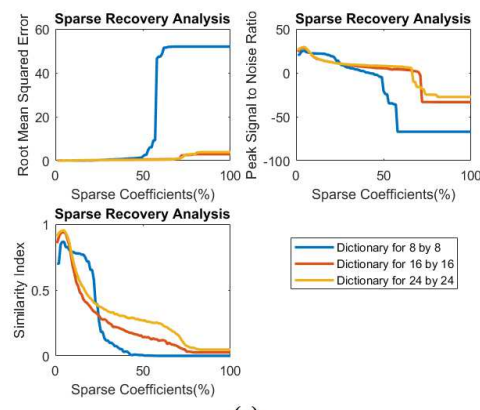

(a)
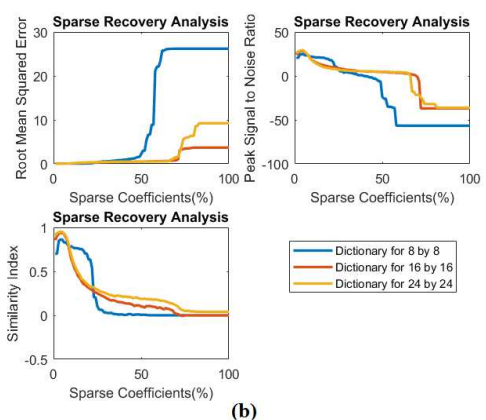

Fig. 6 Image assessment index Vs number of sparse coefficient (a) For random sensing matrix (b) For proposed sensing matrix

\subsection{Compressing and encryption}

The compression of the sparse data is the easiest step in architecture. It is simply multiplying the sparse representation by sensing matrix. Both encryption and compression have been done simultaneously. As long as the sensing matrix is secreted from third-party users, the compressed data remain safe from being attacked by external agents. Furthermore, it can be constructed from the encryption key that already could be obtained from algorithm-I and II. Therefore, there is no need of sending the whole sensing matrix; instead, securely transferring the encryption keys is enough.

\subsection{Decompression and decryption}

This step or simultaneous decompression and decryption is the reverse process of the previous one and occurs at the receiver side. Once the sensing matrix 
keys are delivered to the receiver, the decompression is possible by solving the p-1 optimization, eq.9, problem using the already described prim-dual interiorpoint method followed by sparse recovery.
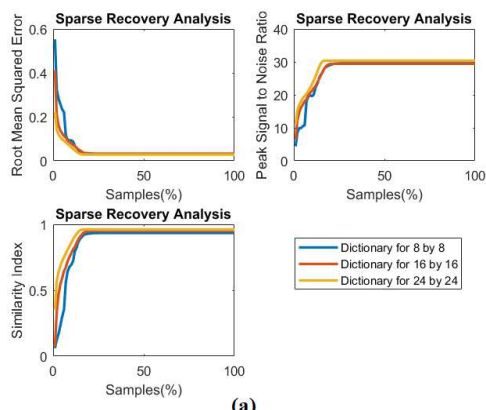
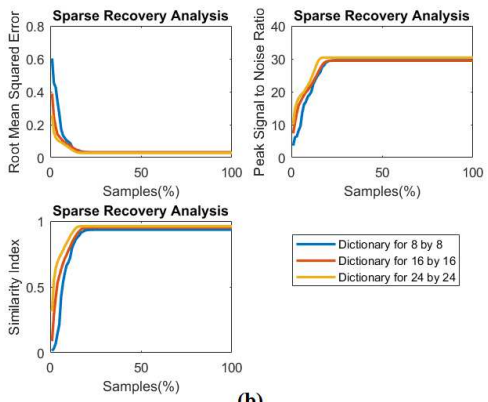

Fig. 7 Image assessment index Vs number of samples (a) for random sensing matrix (b) for proposed sensing matrix

Unlike the previous section, we fix the numbers of the sparse coefficient constant to study the rate of signal recovery with the number of samples taken from the sparse signal. The result shows that still, we have a high recovery rate at a small number of samples. This result is better than the recommended compression rate for wireless transmission, from 20 to $25 \mathrm{~dB}$ [38][39]. Our conclusion from this analysis is, the proposed sensing matrix performance is almost the same as the random matrix, which possesses the RIP feature [40].

\section{Security threat model}

For the safe flow of data from one state to another or from sender to receiver, the communication link should be secured by encrypting the data. This section will start by identifying the expected potential threat that will impact and expose it for risk based on a literature survey.

The critical elements of the system that responsible for the loss of data if they accessed by potential adversaries are the dictionary, the sensing matrix, and the encryption key used to build the sensing matrix. The last element cannot be a problem if they securely transfer to a legitimate user, but the first two elements need further analysis because there are several alternative ways for the attacker to construct them. The dictionary matrix can be constructed using the same algorithm used in the proposed design from different training signals or fingerprint data. However, any output from it may not be sparse simultaneously with the original input data. Or the sparse solution is always unique for the fixed input signal and coherent dictionary matrix once it is 
computed [41]. Hence, we will focus only on the sensing matrix.

When the attacker sent a randomly chosen plane text or image to the oracle and gets back the cipher version of the text or image, there will be a probability for the attacker to gain a piece of knowledge about how the system encrypts its data. This attack is known as Chosen Plain text Attack (CPA). Like other works [42] [43], the proposed encryption in this paper is related but not directly to the sensing matrix. As already pointed out in [44] [45], such a kind of compressed system is not secured against CPA. We can get rid of this vulnerability by effectively extracting different sensing matrices with high probability using the modified LCG (eq. 14) from a single DCT matrix designed based on a chaotic model (eq. 13). And the rest will be discussed in the next section.

\section{Discussion on the performance and reliability of the proposed system}

In this section, we will identify the sensitive part of the system that makes it vulnerable to attack and explain how the system will respond to them by analyzing how the model impacts the reliability of the design approach. We begin by demonstrating the work flow diagramatically in fig. 8 and by insuring the system does't damage most of the fingerprint minutiae by showing that it preservs majority of them in fig.9.
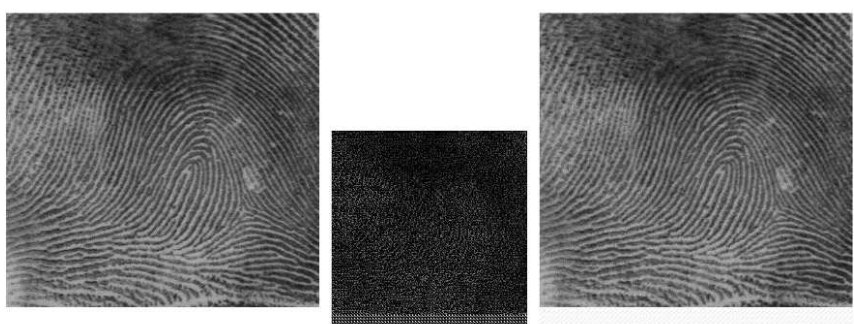

Fig. 8 From left to right, the original fingerprint image, the compressed also encrypted version and the recovered image respectively

\subsection{Randomness test via correlation analysis}

Correlation analysis involves evaluating the correlation of several randomly selected pairs of adjacent pixels aligned horizontally, vertically, and diagonally. For a particular fingerprint image with each pixel coordinates $(x, y)$ selected 


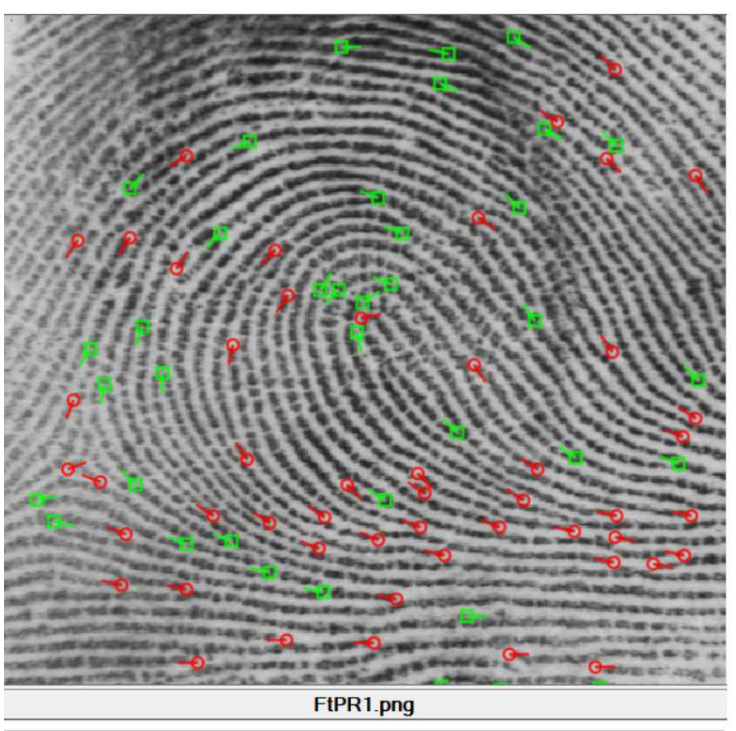

(a)

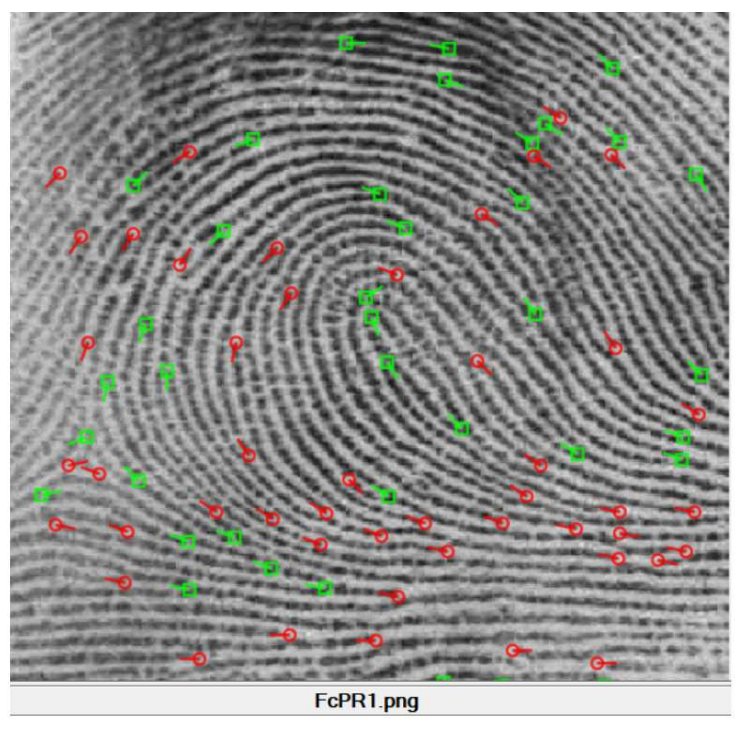

(b)

Fig. 9 Fingerprint minutiae view of (a) transmitted and (b) recovered fingerprint image

randomly, the correlation is given by eq. 18 .

$$
\operatorname{Corr}(x, y)=\frac{T \sum_{i=1}^{T}\left(x_{i} y_{i}\right)-\sum_{i=1}^{T} x_{i} \sum_{i=1}^{T} y_{i}}{\sqrt{\left(T \sum_{i=1}^{T} x_{i}^{2}-\left(\sum_{i=1}^{T} x_{i}\right)^{2}\right)^{2}\left(T \sum_{i=1}^{T} y_{i}^{2}-\left(\sum_{i=1}^{T} y_{i}\right)^{2}\right)^{2}}}
$$

We effectively utilized eq.18 for 3000-pixel pairs of plain and compressed cipher images and plot distribution, as shown in Fig. 10. Comparing the figure in both plain and cipher image shows that the proposed sensing matrix possesses the expected randomness.
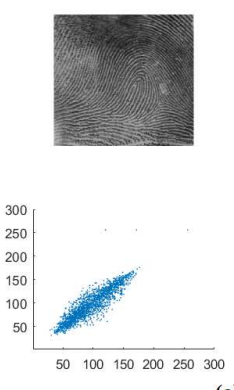

(a)
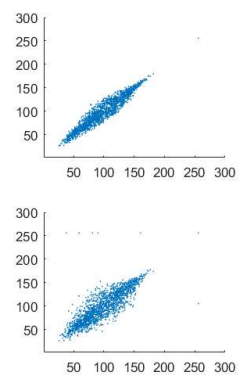
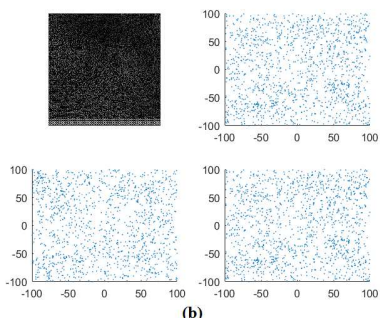

Fig. 10 Correlation plots (a) for plain-image and (b) compressed image 
6.2 Histogram analysis for encrypted image

The histogram analysis is helpful to identify which data of the securely compressed image easily visible for the attacker. In the proposed scheme, there are several entries whose values are negative. Take this as one advantage and urthermore, the nearly uniform distribution of values as depicted on the histogram comparison figure, fig.11 further indicates that the system is still safe from histogram-based attacks.
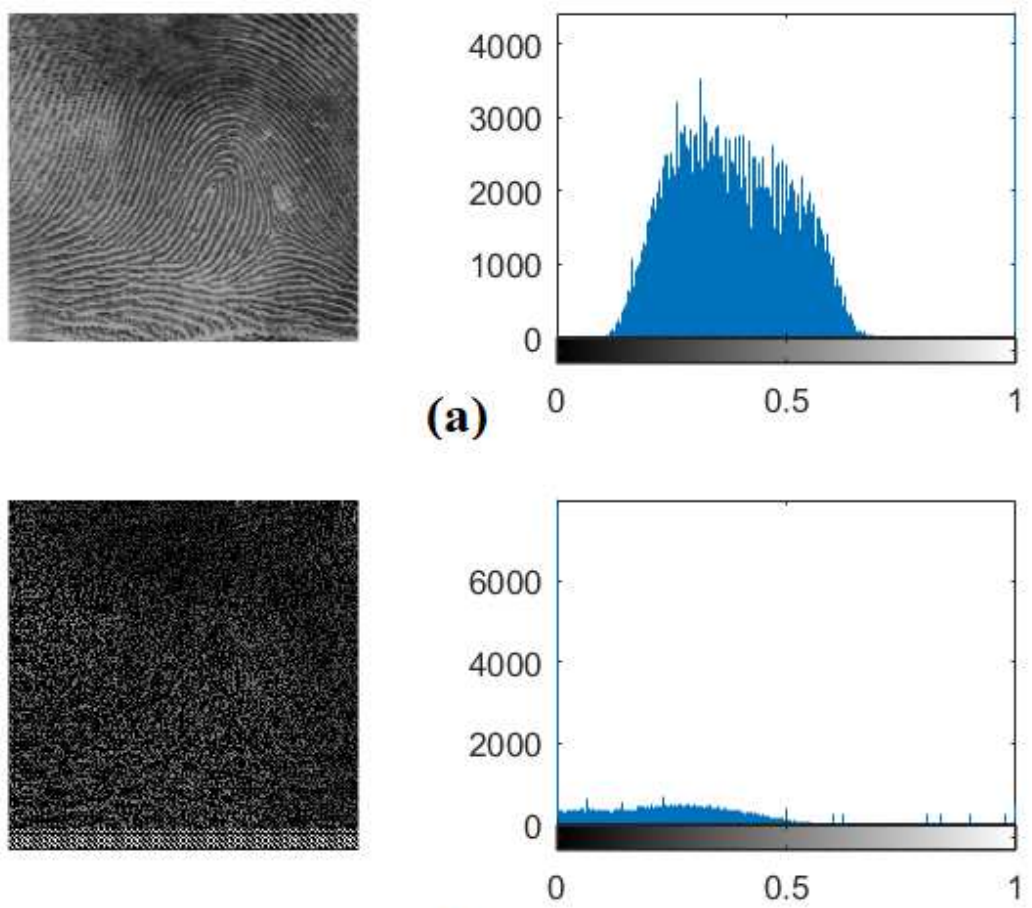

(b)

Fig. 11 Histogram plot for (a) plain-image and (b) compressed figure

\subsection{Key Sensitivity Test}

Key sensitivity test used to examine how the proposed sensing matrix responds to a slight change in the magnitude of some of the keys used to construct the proposed sensing matrix. Keeping other keys that affect others except for the logistic map the same, we have observed how the sensing matrix row sequence 
generated if the growth rate of the Logistic map changed by $2.6333 \times 10^{-6} \%$ with the help of our proposed algorithm-I. The growth rate, which is equal to 3.7504 selects the rows of the DCT matrix according to the sequences of $8,29,2,15,19,12 \ldots$ And the other 3.7505 increase by only $2.6333 \times 10^{-6 \%}$ produces the sequences of $8,28,3,17,19,13 \ldots$ Therefore, the partially orthogonal sensing matrix with those selected rows from DCT produces different cipher data. The same precision has been used in all computations of sequence and initial values. This behaviour of the sensing matrix shows that the proposed scheme is a key sensitive one.

\subsection{Key space of the proposed system}

When a brute force attack occurs, the adversary constructs the sensing matrix by combining rows using its technique with many trials. According to [46] [47], the attacker must attempt a maximum of $2^{100}$ rows combinations of the DCT matrix, which supposed to exceed the keyspace of the system. In this regard, the keyspace of our proposed approach can be computed for row dimension 121 , and 25 of them, which are enough to construct the sensing matrix.

$$
\text { Key Space }=(121 !) \times\left(\frac{121 !}{25 !(121-25) !}\right)=42.55^{255} \cong 2^{1225} \gg 2^{100}
$$

The term in the first bracket is the number of options for an attacker to construct the masked DCT whereas the second matrix is the number of option to construct the sensing matrices and their product give large enough value to turn the brute force attack into infeasible for the proposed system.

\subsection{Differential attack}

Types of attack that we have already studied before requiring analysis involve the whole signal or image at a time. Differential attack analysis more focused on a specific single pixel. Therefore, we only take the image block consists the pixel of our interest because different sensing matrices were used to resist brute force and chosen cipher text treat. Therefore, our analysis will be based on a selected specific block using NPCR (Net Pixel Change Rate) and UACI (Unified Average Changing Intensity), given by eq. 20 and 21.

$$
\begin{gathered}
N C P R=\frac{\sum_{i, j} D(i, j)}{H \times W} \times 100 \% \\
U A C I=\frac{1}{H \times W} \sum_{i, j} \frac{\left|c(i, j)-c^{\prime}(i, j)\right|}{255} \times 100 \%
\end{gathered}
$$

Where $H$ and $W$ are the height and width of the image block. And $c(i, j)$ is the plain image block whereas $c^{\prime}(i, j)$ also the encrypted image of the block 
with one of the pixels changed. The value of $D(i, j)$ is one if there is pixel difference between $c(i, j)$ and $c^{\prime}(i, j)$ otherwise it is zero. Our results for samples taken from eight by eight block is $100 \%$ for NCPR and $0.92 \%$ for UACI. This shows that all sixty-four-pixel values undergo changes that would make the design scheme resistive to any attack.

\subsection{Entropy analysis}

The entropy of our compressed system is the measurement of the system's ability to generate random encrypted output. The entropy $H$ of the image with probability $P$ of total number $N$ is mathematically expressed and given by eq. 22 .

$$
H=\sum_{i=1}^{N} P_{i} \log _{2} P_{i}
$$

Since our system designed based on the $D C T$ matrix, there are high numbers of entries whose values are negative. Hence to compute the logarithmic content of equation (22), we took the absolute value of the cipher image, and we have verified that the system has 7.20 , which is $90 \%$ of the ideal value.

\section{Conclusion}

In this work, we aimed at a secured transmission of fingerprint images using a compressive sensing approach. To establish security, we have designed a novel deterministic sensing matrix based on a partial orthogonal matrix derived from a Discrete Cosine Transform (DST) matrix. A modified multiplicative linear congruential generator $(M L C G)$ has been employed to construct the sensing matrix in a deterministic manner from the $D C T$ matrix. The added shifting factor in the $M L C G$ expression enables us to use any rows of the $D C T$ matrix to build our sensing matrix. The traditional $M L C G$ has limitations in choosing any of the rows until the maximum row index of the $D C T$ matrix. By effectively applying the shifting factor, we successfully select any rows entry per the requirement of the sub-matrix to be used as a measurement matrix. The comprehensive simulation-based analysis of our proposed compressed sensing system shows that the deterministic sensing matrix has better performance than the non-deterministic or random matrix in terms of the number of sensors that effectively used. And this may open a new way for the quest of an optimized alternative fingerprint scanning technology.

\section{Conflict of interest}

The authors declare that they have no conflict of interest. 


\section{References}

1. F. Liu, D. Zhang, 3D fingerprint reconstruction system using feature correspondences and prior estimated finger model, Pattern Recognit. 47 (2014) 178-193. https://doi.org/10.1016/j.patcog.2013.06.0+09.

2. M.K. Thakar, T. Sharma, Digital grid method for fingerprint identification and objective report writing, Egypt. J. Forensic Sci. 6 (2016) 194-201. https://doi.org/10.1016/j.ejfs.2016.05.008.

3. M. Lastra, J. Carabaño, P.D. Gutiérrez, J.M. Benítez, F. Herrera, Fast fingerprint identification using GPUs, Inf. Sci. (Ny). $301 \quad$ (2015) 195-214. https://doi.org/10.1016/j.ins.2014.12.052.

4. N. Sato, S. Shigematsu, H. Morimura, M. Yano, K. Kudou, T. Kamei, K. Machida, Novel surface structure and its fabrication process for MEMS fingerprint sensor, IEEE Trans. Electron Devices. 52 (2005) 1026-1032. https://doi.org/10.1109/TED.2005.846342.

5. M.A.U. Khan, T.M. Khan, D.G. Bailey, Y. Kong, A spatial domain scar removal strategy for fingerprint image enhancement, Pattern Recognit. 60 (2016) 258-274. https://doi.org/10.1016/j.patcog.2016.05.015.

6. S. Kim, B. Park, B.S. Song, S. Yang, Deep belief network based statistical feature learning for fingerprint liveness detection, Pattern Recognit. Lett. 77 (2016) 58-65. https://doi.org/10.1016/j.patrec.2016.03.015.

7. E.J. Candès, J. Romberg, T. Tao, Robust uncertainty principles: Exact signal reconstruction from highly incomplete frequency information, IEEE Trans. Inf. Theory. (2006). https://doi.org/10.1109/TIT.2005.862083.

8. D.L. Donoho, Compressed sensing, IEEE Trans. Inf. Theory. (2006). https://doi.org/10.1109/TIT.2006.871582.

9. E.J. Candès, D.L. Donoho, New tight frames of curvelets and optimal representations of objects with piecewise C 2 singularities , Commun. Pure Appl. Math. 57 (2004) 219-266. https://doi.org/10.1002/cpa.10116.

10. J.B. Allen, L.R. Rabiner, A Unified Approach to Short-Time Fourier Analysis and Synthesis, Proc. IEEE. 65 (1977) 1558-1564. https://doi.org/10.1109/PROC.1977.10770.

11. G. Chen, M. Maggioni, Multiscale geometric wavelets for the analysis of point clouds, 2010 44th Annu. Conf. Inf. Sci. Syst. CISS 2010. (2010) 1-6. https://doi.org/10.1109/CISS.2010.5464843.

12. M. Aharon, M. Elad, A. Bruckstein, K-SVD: An algorithm for designing overcomplete dictionaries for sparse representation, IEEE Trans. Signal Process. 54 (2006) 4311-4322. https://doi.org/10.1109/TSP.2006.881199.

13. J. Mairal, F. Bach FRANCISBACH, J. Ponce JEANPONCE, G. Sapiro, Online Learning for Matrix Factorization and Sparse Coding, J. Mach. Learn. Res. 11 (2010) 19-60. http://www.jmlr.org/papers/volume11/mairal10a/mairal10a.pdf.

14. K. Kreutz-delgado, J.F. Murray, T.J. Sejnowski, Dictionary Learning Algorithms for Sparse Representation Kenneth, Neural Comput. 15 (2003). https://doi.org/10.1162/089976603762552951.

15. J. Mairal, F. Bach, J. Ponce, G. Sapiro, Online learning for matrix factorization and sparse coding, J. Mach. Learn. Res. (2010). https://doi.org/10.1145/1756006.1756008.

16. I. Tawfic, S. Kayhan, Compressed sensing of ECG signal for wireless system with new fast iterative method, Comput. Methods Programs Biomed. (2015). https://doi.org/10.1016/j.cmpb.2015.09.010.

17. L.F. Polania, R.E. Carrillo, M. Blanco-Velasco, K.E. Barner, Exploiting prior knowledge in compressed sensing wireless ECG systems, IEEE J. Biomed. Heal. Informatics. (2015). https://doi.org/10.1109/JBHI.2014.2325017.

18. D. Liu, Q. Wang, Y. Zhang, X. Liu, J. Lu, J. Sun, FPGA-based real-time compressed sensing of multichannel EEG signals for wireless body area networks, Biomed. Signal Process. Control. 49 (2019) 221-230. https://doi.org/10.1016/j.bspc.2018.12.019.

19. Ö. Polat, S.K. Kayhan, High-speed FPGA implementation of orthogonal matching pursuit for compressive sensing signal reconstruction, Comput. Electr. Eng. 71 (2018) 173-190. https://doi.org/10.1016/j.compeleceng.2018.07.017.

20. M. Kharratzadeh, A. Sharifnassab, M. Babaie-Zadeh, Invariancy of Sparse Recovery Algorithms, IEEE Trans. Inf. Theory. $63 \quad$ (2017) 3333-3347. https://doi.org/10.1109/TIT.2017.2686428. 
21. D. Gangopadhyay, E.G. Allstot, A.M.R. Dixon, K. Natarajan, S. Gupta, D.J. Allstot, Compressed sensing analog front-end for bio-sensor applications, IEEE J. Solid-State Circuits.(2014). https://doi.org/10.1109/JSSC.2013.2284673.

22. B. Javidi, Optical encryption using a joint transform correlator architecture, Opt. Eng. (2000). https://doi.org/10.1117/1.1304844.

23. B. Javidi, Noise performance of double-phase encryption compared to XOR encryption, Opt. Eng. (1999). https://doi.org/10.1117/1.602074.

24. Y. Zhang, C.H. Zheng, N. Tanno, Optical encryption based on iterative fractional Fourier transform, Opt. Commun. (2002). https://doi.org/10.1016 S0030-4018(02)011136.

25. M.N. Islam, Encryption and multiplexing of fingerprints for enhanced security, 2011 IEEE Long Isl. Syst. Appl. Technol. Conf. LISAT 2011. (2011) 0-3. https://doi.org/10.1109/LISAT.2011.5784235.

26. G. Mehta, M.K. Dutta, J. Karasek, P.S. Kim, An efficient and lossless fingerprint encryption algorithm using Henon map \& Arnold transformation, 2013 Int. Conf. Control Commun. Comput. ICCC 2013. (2013) 485-489. https://doi.org/10.1109/ICCC.2013.6731703.

27. M. Vidyasagar, A tutorial introduction to compressed sensing, 2016 IEEE 55th Conf. Decis. Control. CDC 2016. (2016) 5091-5104. https://doi.org/10.1109/CDC.2016.7799048.

28. Y.C. Pati, R. Rezaiifar, P.S. Krishnaprasad, Orthogonal matching pursuit: recursive function approximation with applications to wavelet decomposition, in: Conf. Rec. Asilomar Conf. Signals, Syst. Comput., 1993. https://doi.org/10.1109/acssc.1993.342465.

29. [29] S. Mallat, A Wavelet Tour of Signal Processing, 2009. https://doi.org/10.1016/B978-0-12- 374370-1.X0001-8.

30. A. Ravelomanantsoa, H. Rabah, A. Rouane, Compressed Sensing: A Simple Deterministic Measurement Matrix and a Fast Recovery Algorithm, IEEE Trans. Instrum. Meas. 64 (2015) 3405-3413. https://doi.org/10.1109/TIM.2015.2459471.

31. H. Djelouat, A. Amira, F. Bensaali, I. Boukhennoufa, Secure compressive sensing for ECG monitoring, Comput. Secur. 88 (2020) 101649. https://doi.org/10.1016/j.cose.2019.101649.

32. R.M. May, Simple mathematical models with very complicated dynamics, Nature. (1976). https://doi.org/10.1038/261459a0.

33. D. Ruelle, F. Takens, On the nature of turbulence, Commun. Math. Phys. (1971). https://doi.org/10.1007/BF01646553.

34. S.G. Mallat, Z. Zhang, Matching Pursuits With Time-Frequency Dictionaries, IEEE Trans. Signal Process. (1993). https://doi.org/10.1109/78.258082.

35. D. Needell, J.A. Tropp, CoSaMP: Iterative signal recovery from incomplete and inaccurate samples, Appl. Comput. Harmon. Anal. (2009). https://doi.org/10.1016/j.acha.2008.07.002.

36. M. Aharon, M. Elad, A.M. Bruckstein, On the uniqueness of overcomplete dictionaries, and a practical way to retrieve them, Linear Algebra Appl. (2006). https://doi.org/10.1016/j.laa.2005.06.035.

37. Z. Wang, A.C. Bovik, H.R. Sheikh, E.P. Simoncelli, Image quality assessment: From error visibility to structural similarity, IEEE Trans. Image Process. (2004). https://doi.org/10.1109/TIP.2003.819861.

38. E.J. Candès, The restricted isometry property and its implications for compressed sensing, Comptes Rendus Math. (2008). https://doi.org/10.1016/j.crma.2008.03.014.

39. L. Chao, J. Han, L. Yan, L. Sun, F. Huang, Z.B. Zhu, S. Wei, H. Ji, D. Ma, Fast compressed sensing analysis for imaging reconstruction with primal dual interior point algorithm, Opt.Lasers Eng. 129 (2020). https://doi.org/10.1016/j.optlaseng.2020.106082.

40. G.S. Fishman, L.R. Moore III, Erratum: An Exhaustive Analysis of Multiplicative Congruential Random Number Generators with Modulus $2^{31}-1$, SIAM J. Sci. Stat. Comput. (1986). https://doi.org/10.1137/0907072.

41. M. Bakiri, C. Guyeux, J.F. Couchot, A.K. Oudjida, Survey on hardware implementation of random number generators on FPGA: Theory and experimental analyses, Comput. Sci. Rev. 27 (2018) 135-153. https://doi.org/10.1016/j.cosrev.2018.01.002.

42. N. Thomos, N. V. Boulgouris, M.G. Strintzis, Optimized transmission of JPEG2000 streams over wireless channels, IEEE Trans. Image Process. (2006). https://doi.org/10.1109/TIP.2005.860338. 
43. X. Li, J. Cai, Robust transmission of JPEG2000 encoded images over packet loss channels, in: Proc. 2007 IEEE Int. Conf. Multimed. Expo, ICME 2007, 2007. https://doi.org/10.1109/icme.2007.4284808.

44. M. Elad, A.M. Bruckstein, A generalized uncertainty principle and sparse representation in pairs of bases, IEEE Trans. Inf. Theory. (2002). https://doi.org/10.1109/TIT.2002.801410.

45. S.A. Hossein, A.E. Tabatabaei, N. Zivic, Security analysis of the joint encryption and compressed sensing, in: 2012 20th Telecommun. Forum, TELFOR 2012 - Proc., 2012. https://doi.org/10.1109/TELFOR.2012.6419328.

46. A. Orsdemir, H.O. Altun, G. Sharma, M.F. Bocko, On the security and robustness of encryption via compressed sensing, in: Proc. - IEEE Mil. Commun. Conf. MILCOM, 2008. https://doi.org/10.1109/MILCOM.2008.4753187.

47. G. Hu, D. Xiao, Y. Wang, T. Xiang, An image coding scheme using parallel compressive sensing for simultaneous compression-encryption applications, J. Vis. Commun. Image Represent. 44 (2017) 116-127. https://doi.org/10.1016/j.jvcir.2017.01.022.

48. Y. Zhang, L.Y. Zhang, J. Zhou, L. Liu, F. Chen, X. He, A Review of Compressive Sensing in Information Security Field, IEEE Access. (2016). https://doi.org/10.1109/ACCESS.2016.2569421.

49. G. Alvarez, S. Li, Some basic cryptographic requirements for chaos-based cryptosystems, Int. J. Bifurc. Chaos. (2006). https://doi.org/10.1142/S0218127406015970.

50. A.A. Shah, S.A. Parah, M. Rashid, M. Elhoseny, Efficient image encryption scheme based on generalized logistic map for real time image processing, J. Real-Time Image Process. 17 (2020) 2139-2151. https://doi.org/10.1007/s11554-020-01008-4. 